\title{
Immunoglobulin therapy ameliorates the phenotype and increases lifespan in the severely affected dystrophin-utrophin double knockout mice
}

\author{
Bruno Ghirotto Nunes ${ }^{1}$ - Flávio Vieira Loures ${ }^{2} \cdot$ Heloisa Maria Siqueira Bueno ${ }^{1}$ Erica Baroni Cangussu ${ }^{1}$. \\ Ernesto Goulart $^{1} \cdot$ Giuliana Castello Coatti $^{1} \cdot$ Elia Garcia Caldini $^{3} \cdot$ Antonio Condino-Neto $^{2} \cdot$ Mayana Zatz $^{1}$
}

Received: 4 May 2017 / Revised: 13 September 2017 / Accepted: 14 September 2017 / Published online: 27 October 2017

(C) European Society of Human Genetics 2017

\begin{abstract}
Duchenne muscular dystrophy (DMD) is an X-linked recessive disorder, caused by mutations in the dystrophin gene, affecting 1:3500-5000 boys worldwide. The lack of dystrophin induces degeneration of muscle cells and elicits an immune response characterized by an intensive secretion of pro-inflammatory cytokines. Immunoglobulins modulate the inflammatory response through several mechanisms and have been widely used as an adjuvant therapy for autoimmune diseases. Here we evaluated the effect of immunoglobulin G (IG) injected intraperitoneally in a severely affected double knockout (dko) mouse model for Duchenne muscular dystrophy. The IG dko treated mice were compared regarding activity rates, survival and histopathology with a control untreated group. Additionally, dendritic cells and naïve lymphocytes from these two groups and WT mice were obtained to study in vitro the role of the immune system associated to DMD pathophysiology. We show that IG therapy significantly enhances activity rate and lifespan of dko mice. It diminishes muscle tissue inflammation by decreasing the expression of costimulatory molecules MHC, CD86 and CD40 and reducing Th1-related cytokines IFN- $\gamma$, IL-1 $\beta$ and TNF- $\alpha$ release. IG therapy dampens the effector immune responses supporting the hypothesis according to which the immune response accelerates DMD progression. As IG therapy is already approved by FDA for treating autoimmune disorders, with less side-effects than currently used glucocorticoids, our results may open a new therapeutic option aiming to improve life quality and lifespan of DMD patients.
\end{abstract}

\section{Introduction}

Duchenne muscular dystrophy (DMD) is a genetic neuromuscular disorder caused by mutations in the DMD gene (at

Electronic supplementary material The online version of this article (https://doi.org/10.1038/s41431-017-0017-y) contains supplementary material, which is available to authorized users.

Antonio Condino-Neto

condino@icb.usp.br

$\triangle$ Mayana Zatz

mayazatz@usp.br

1 Human Genome and Stem-Cell Research Center, Institute of Biosciences, University of São Paulo, Sao Paulo, SP, Brazil

2 Department of Immunology, Institute of Biomedical Sciences, University of São Paulo, Sao Paulo, SP, Brazil

3 Department of Pathology, School of Medicine, University of São Paulo, Sao Paulo, SP, Brazil
Xp21.2) resulting in muscle dystrophin deficiency. DMD is the commonest progressive form of muscular dystrophy (PMD) affecting 1 in 3500-5000 males worldwide. The clinical symptoms usually become apparent at age 3-5 years, with difficulties for jumping, running or rising from the floor. Upper limbs are progressively affected and most patients' loss of ambulation occurs around 10-12 years of age. Death from cardiopulmonary complications usually occurs in the second or third decade [1]. Aiming to treat DMD, several pharmacological and more recently gene therapy approaches have been tested over the years [2-4]. Despite numerous efforts, only glucocorticoid therapy, which has been replicated in different studies, slows down the progression of the disease. Their beneficial effects have been explained by their immunosuppressive properties. Nevertheless, the continued use of these drugs brings many side effects including obesity, hypertension and behavioral changes among other features [5].

Patients carrying nonsense DMD mutations are unable to produce a functional isoform of dystrophin, a large $427 \mathrm{kD}$ 
cytoskeletal protein located in the sarcolemma of skeletal muscles, responsible for the structural stabilization of the myofibers plasma membrane, giving them resistance to muscle contraction and relaxation cycles and strength maintenance. The lack of the protein in myofibers results in severe muscle degeneration and weakness, replacement of muscle by fat, connective tissue, and premature death in affected patients [1]. Due to the structural instability of the sarcolemma, there is a release into the extracellular medium of several components such as creatine phosphokinase (CPK), ATP, damage-associated molecular patterns (DAMPs) and mRNAs. ATP binds to purinergic receptor P2X7 inducing muscle cell death whereas DAMPs interact with toll-like receptors (TLRs), initiating an innate immune response through activation of the $\mathrm{NF}-\kappa \mathrm{B}$ signaling pathway, leading to a chronic inflammatory condition in muscle tissue, accumulation of proinflammatory cytokines, activation of dendritic cells, macrophages, and eventually the adaptive immune response through $\mathrm{CD}^{+}{ }^{+}$and $\mathrm{CD}^{+} \mathrm{T}$ cells $[6,7]$.

Immunoglobulins are glycoproteins secreted by plasmatic cells, the active form of B lymphocytes as part of the adaptive immune response. Immunoglobulin $\mathrm{G}$ therapy (IG therapy) has been used as a replacement therapy for patients with antibody deficiency or as a modulatory adjuvant therapy for patients with autoimmune or inflammatory diseases such as immunothrombocytopenia (ITP), GuillainBarré syndrome (GBS), Chronic Inflammatory Demyelinating Polyneuropathy (CIDP), Kawasaki syndrome (KS) and Systemic Lupus Erythematosus (SLE) [8]. Modulatory IG therapy mechanisms of action include blockade of Fc receptors dependent pathways, saturation of FcRn receptors on endothelial cells, neutralization of cytokines and autoantibodies by anti-idiotypic antibodies, and scavenging of the complement system anaphylatoxins $\mathrm{C} 3 \mathrm{a}$ and C5a [9-12].

Considering the inflammatory nature of DMD in humans, the side effects related to glucocorticoid therapy, the modulatory properties of IG therapy for other inflammatory diseases [12-14] and its potential benefits for patients with DMD, our aim was to evaluate the potential clinical effects of human polyclonal IgG delivered intraperitoneally in the severely affected dko mdx/utr- mice, focusing on $\operatorname{IgG}$ immunomodulatory properties in treated animals' dystrophic muscle.

\section{Materials and methods}

\section{Animals, ethics and experimental protocol}

This study was approved by the ethics committee of animals' experiments at Institute of Biosciences, University of
São Paulo (protocol number CEUA-IBUSP 260/2016). Double knockout (dko) mice (Utrn (tm1Jrs) Dmd (mdx)/ J), The Jackson Laboratory's - USA) were divided into two groups: (a) the IG treated group received a dosage of $2 \mathrm{~g}$ / mouse $\mathrm{kg}$ of human polyclonal immunoglobulin $\mathrm{G}$ intraperitoneally (Kiovig 10\%, Baxter, USA), and (b) the control group received an equivalent volume of standard injectable saline solution. The animals were injected once a month, starting at 4 weeks of age. Those used for histology and immunology experiments were given 2 IG injections, being sacrificed 20 days after the second injection (Fig. S1).

\section{Survival rate}

Dko IG treated and control mice groups were followed daily. Each animal death and corresponding ages were computed for subsequent statistical analysis. Survival was analyzed by Kaplan Meier curves using the statistical software GraphPad Prism 5 and the data obtained from observations of the animals were analyzed using the logrank test (Mantel Cox) and Gehan-Beslow-Wilcoxon (GBW), with statistical significance of $p<0.05$.

\section{Activity rate}

To measure activity rate, the treadmill test was used, starting with a speed of $2 \mathrm{~m} / \mathrm{min}$ and increasing $1 \mathrm{~m} / \mathrm{min}$ at each completed minute. A shock stimulus in the treadmill was used to keep the animals running. By touching the mat background each animal was removed, placed back in its cage and the running time was recorded. The average group activity was calculated every day by adding the running time of each mouse and dividing by the total number of animals. The mean activity rate calculated from the experimental data from GraphPad Prism 5 was analyzed with an unpaired two-tailed Student's t test, with a significance level of $p<0.05$.

\section{Muscle histology}

To analyze the muscle inflammatory state, the animals were euthanized 20 days after receiving the second IG injection or saline in the control group. Quadriceps, gastrocnemius and diaphragm were extracted from treated and control mice and fixed with $4 \%$ paraformaldehyde (Sigma). The material was dehydrated in ethanol and xylol, processed for paraffin embedment, sliced in a microtome and submitted to standard staining protocol by Hematoxylin and Eosin (H\&E) and Picrosirius counterstained with Hematoxylin (PSH). PSH stained slides were pictured using polarized light that evidences thick collagen fibers in red.

Histopathological quantification analyses of gastrocnemius, quadriceps and diaphragm included three 


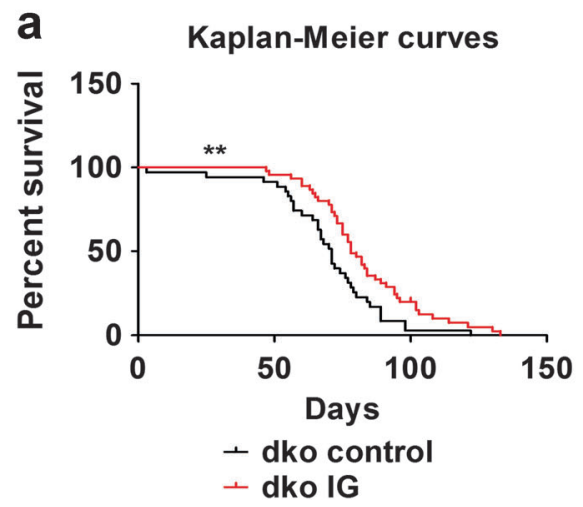

Fig. 1 Animal survival and physical assessment. a Kaplan Meier curves show that IG treatment increases significantly the lifespan of a group of 45 dko IG treated mice (being 25 females and 20 males) as compared to 35 untreated controls (being 21 females and 14 males),

parameters: percentage of inflammatory clusters and thick collagen fibers and the central nucleation index of the fibers per slice. Analyses were performed using Image $\mathbf{J}$ software for $n=6$ mice ( 3 male dko controls and 3 dko IG treated mice, 2 being females and 1 male). For each animal, a total of four images per muscle slice were evaluated and the mean value was determined. For statistical analysis, the mean of each animal was considered. Images were analyzed at $100 \times$ magnification, which is the power of the objective lens $(20 \times)$ multiplied by the magnification power of the ocular lens $(5 \times)$. When present in the slices, blank areas were measured and subtracted from the total area.

\section{DC generation and maturation in vitro}

Bone-marrow derived DCs (BMDCs) were generated according to previously described methods (Inaba et al. [15]) with some modifications. Briefly, cells removed from femurs of WT, dko control and dko IG treated mice were cultured in low-attachment cell-plates with $20 \mathrm{ng} / \mathrm{mL}$ recombinant granulocyte-macrophage colony-stimulating factor (rGM-CSF; BD Bioscience; San Jose, CA, USA) and $2 \mathrm{ng} / \mathrm{mL}$ interleukin-4 (rIL-4; BD Bioscience) in complete medium RPMI (Difco, Detroit, MI, USA) containing $10 \%$ fetal calf serum, $2 \mathrm{mM}$ L-glutamine, $100 \mathrm{U} / \mathrm{mL}$ penicillin, and $100 \mu \mathrm{g} / \mathrm{mL}$ streptomycin (Sigma, Germany). The medium containing rGM-CSF and rIL-4 was renewed on days 3 and 5 of culture (Fig. S1).

\section{Generation of muscle antigens}

Muscle homogenates were obtained from quadriceps, gastrocnemius and diaphragm of dko IG treated mice, extracted and processed with PBS $1 \%$, until the material became homogeneous. Then, the homogenates were preserved at $-80{ }^{\circ} \mathrm{C}$ until experiments and diluted at a $1: 10$ proportion in

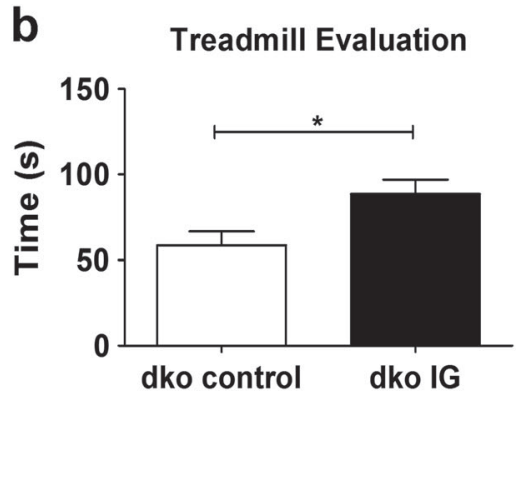

$* * p=0.0080$. $\mathbf{b}$ The treadmill test shows that the average activity rate (means $\pm \mathrm{SE}$ ) is increased in a group of $17 \mathrm{IG}$ treated mice (being 11 males and 6 females) as compared to 15 untreated controls (being 12 females and 3 males), $* p=0.0165$

PBS, with $500 \mu \mathrm{L}$ of the diluted homogenate (used as antigen) being placed in each well to activate the dendritic cells (Fig. S1).

\section{Immune cells analyses}

For cell-surface staining, leukocytes were washed and resuspended at $1 \times 10^{6}$ cells $/ \mathrm{mL}$ in staining buffer (PBS, $2 \%$ fetal calf serum and $0.1 \% \mathrm{NaN} 3$ ). Fc receptors were blocked by the addition of unlabeled anti-CD16/32 (Fc block; BD Biosciences). Leukocytes were then stained in the dark for $20 \mathrm{~min}$ at $4{ }^{\circ} \mathrm{C}$ with the optimal dilution for each monoclonal antibody: PE-Cy7 labeled anti-CD11c; PE anti-MHC, Pacific Blue (PB) labeled anti-CD86, FITC labeled anti-CD40 (from BD Biosciences or BioLegend). Cells were washed twice with staining buffer, fixed with $2 \%$ paraformaldehyde (Sigma) and acquired using a FACSCanto II equipment and FACSDiva software (BD Biosciences). A minimum of 50,000 events were acquired on FACScanto II flow cytometer (BD Biosciences) using the FACSDiva software (BD Biosciences). DCs were gated as judged from forward and side light scatter. The analyses were performed using FlowJo software (Tree Star).

\section{Cytokines profile}

Bone-marrow derived DCs from wild-type and double knockout mice were incubated with muscle homogenates from dko IG treated mice. After $18 \mathrm{~h}$, the supernatants were removed and stored at $-80^{\circ} \mathrm{C}$. Then, DCs were co-cultured with splenic naïve lymphocytes from WT, dko control and dko IG treated mice. The DC:lymphocyte ratio was 1:10. After 6 days the supernatants of DC-lymphocytes co-cultures were removed and stored. The levels of IL- $1 \beta, \mathrm{TNF}-\alpha$, IL-2 and IFN- $\gamma$ were measured by capture enzyme-linked immunosorbent assay (ELISA). The ELISA procedure was 

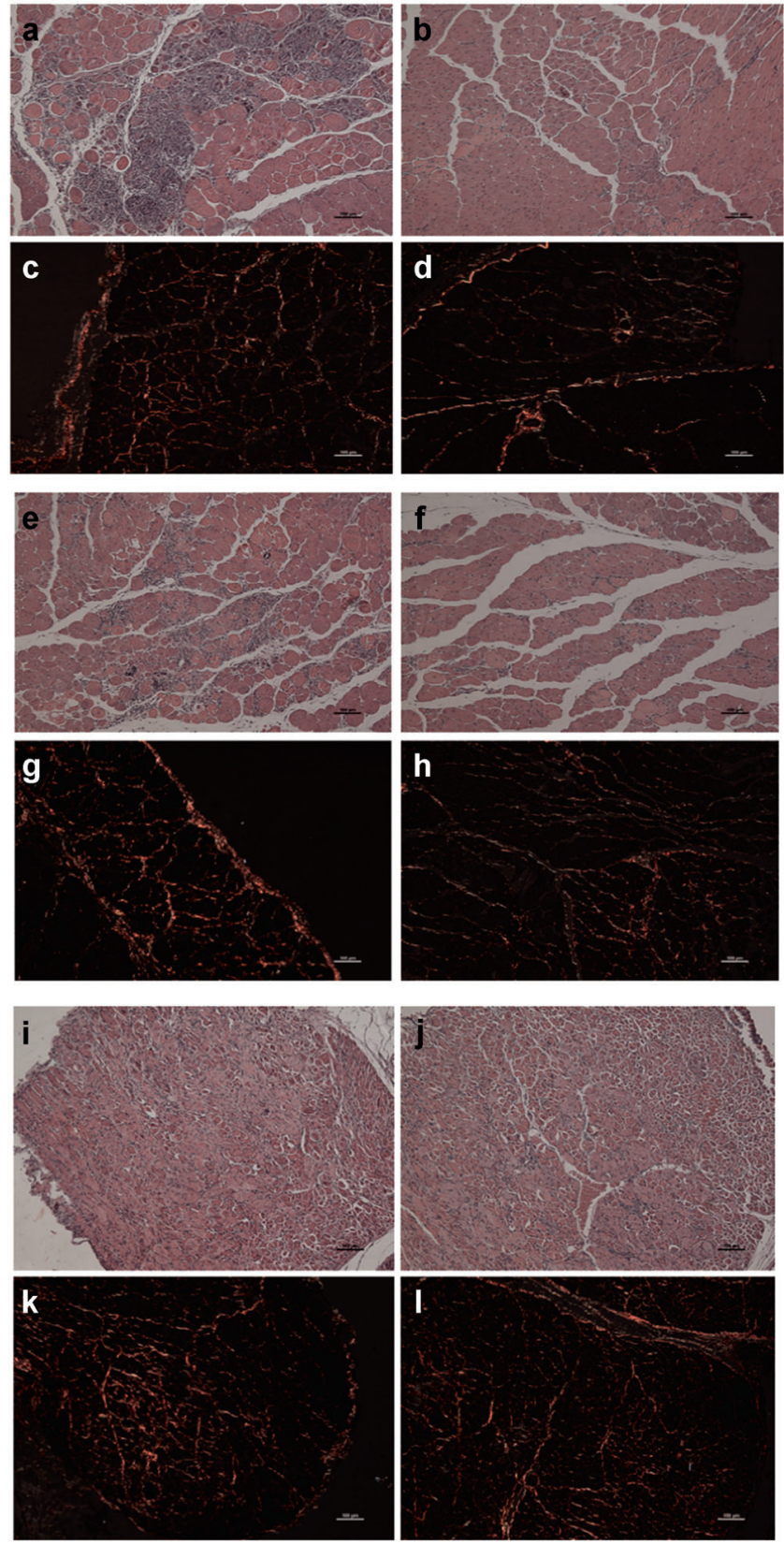

Fig. 2 IG therapy dampens inflammation, fibrosis and suggests regeneration in muscle tissue. $\mathrm{H} \& \mathrm{E}$ staining shows that IG treatment decreases muscle inflammation and lesion and suggests muscle regeneration and PSH staining indicates less collagen fibers deposition in IG treated muscles. Panels $\mathbf{a}-\mathbf{d}$ are from quadriceps muscle (a and $\mathbf{b}$ are H\&E staining, control and IG; $\mathbf{c}$ and $\mathbf{d}$ are PSH staining, control and IG, respectively). Panels $\mathbf{e}-\mathbf{h}$ are from gastrocnemius muscle (e and $\mathbf{f}$ are H\&E staining, control and IG; $\mathbf{g}$ and $\mathbf{h}$ are PSH staining,

performed according to the manufacturer's protocol (Biolegend or eBioscience), and absorbance was measured with a Versa Max Microplate Reader (Molecular Devices; Sunnyvale, CA, USA).
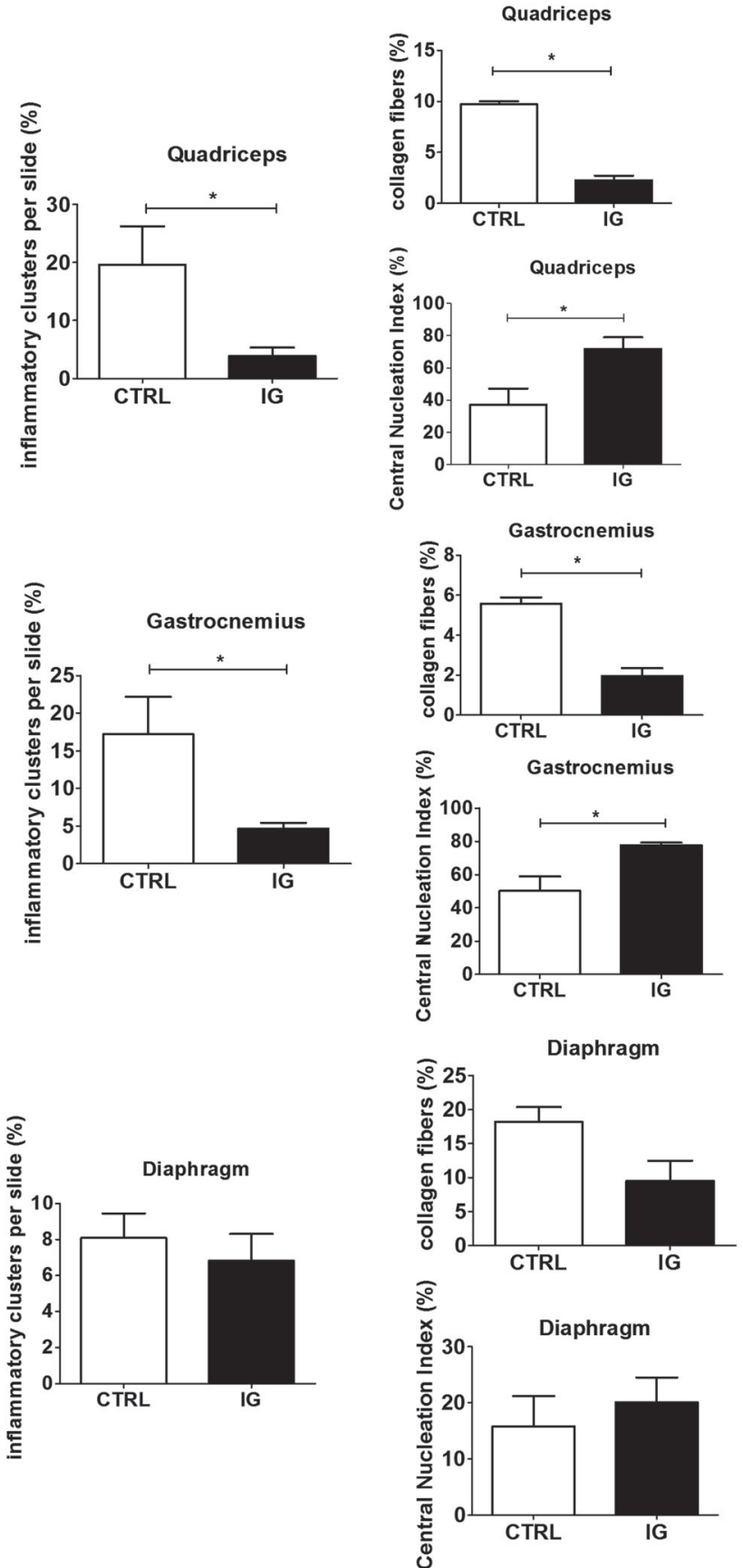

control and IG, respectively). Panels $\mathbf{i}-\mathbf{l}$ are from diaphragm muscle (i and $\mathbf{j}$ are H\&E staining, control and IG; $\mathbf{k}$ and $\mathbf{l}$ are PSH staining, control and IG, respectively), scale bar $=100 \mu \mathrm{m}, 20 \times$ magnitude for all images. For each muscle, there are three graphs in sequence assessing muscle quantification: percentage of inflammatory clusters; percentage of collagen fibers deposition within the tissue and the central nucleation index, respectively; $* p<0.05$

\section{Statistical analyses}

For muscle histology quantification, non-parametric tests (Mann-Whitney) were performed to compare control and 
a

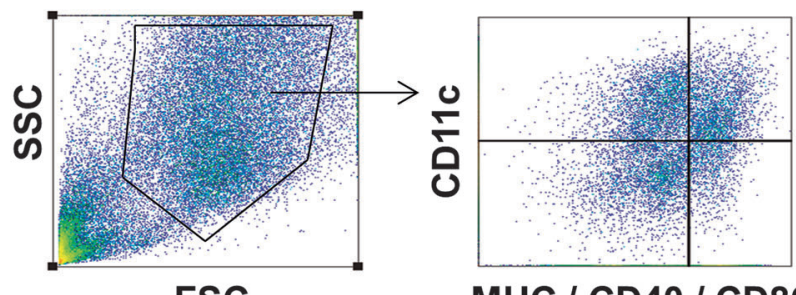

FSC

MHC / CD40 / CD86

b
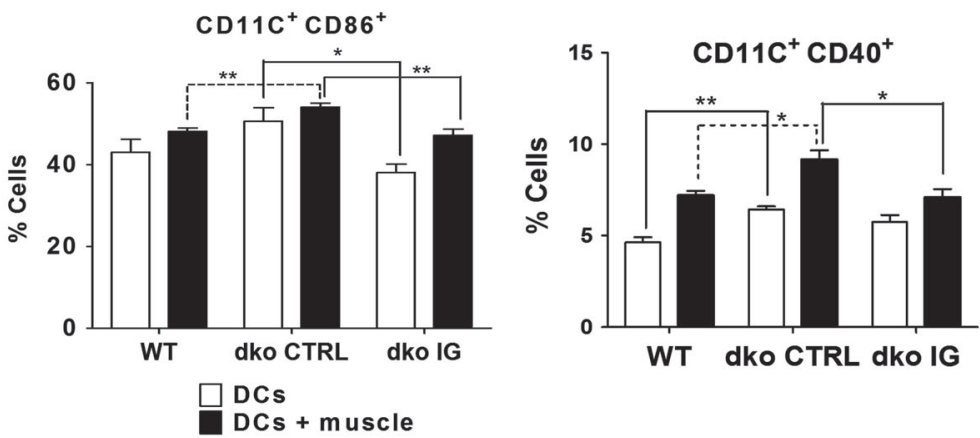

Fig. 3 Immunoglobulin modulates dendritic cell activation profile a Gate strategy used for flow cytometry analysis, with CD11c chosen as a marker for dendritic cell subpopulation and combined with the markers in interest to assess costimulatory molecules expression on DCs surface. b Flow cytometry shows decreased expression of costimulatory molecules on activated DC surface $\left(p=0.0094\right.$ for $\mathrm{MHC}^{+}$;

$p=0.0091$ for $\mathrm{CD}^{+} 6^{+}$and $p=0.0213$ for CD40) in IG treated compared to control mice, data are means \pm SE of six animals per group (WT, dko control and dko IG) from three independent experiments ( $\mathrm{n}=2$ mice per group in each one of them), totalizing $\mathrm{n}=18$ mice analyzed $(* p<0.05 ; * * p<0.01)$

IG mice. One-way ANOVA was performed for both immune cells and cytokines profile analyses, with significance level of $p<0.05$.

\section{Results}

\section{IG treatment increases lifespan and activity rate of dko IG treated mice}

As illustrated in Fig. 1a, Kaplan Meier survival curves show that IG treated animals ( $n=45,25$ being females and 20 males) (red curve) survived significantly more than the control group ( $n=35,21$ being females and 14 males) (black curve). Data was statistically significant for both tests: $p=0.0080$ for the Log Rank test and $p=0.0052$ for the GBW test. The average survival over the period was around 68 days for the control group and 84 days $(\sim 20 \%$ longer $)$ for the $\mathrm{IG}$ treated group.

Additionally, treadmill racing time (Fig. 1b) was significantly higher in the IG group ( $n=17,11$ being males and 6 females), as compared to control group ( $n=15,12$ being females and 3 males), $p=0.0165$. This result indicates that IG treatment increases mice activity rate as well.

\section{Comparative muscle histology in IG treated vs. control groups}

Muscle histology (Fig. 2) was analyzed through standard $\mathrm{H} \& \mathrm{E}$ and $\mathrm{PSH}$ staining to investigate the in situ inflammatory process in quadriceps (Figs. 2a-d), gastrocnemius (Figs. 2e-h) and diaphragm (Figs. 2i-l), from dko controls (2a, 2c, 2e, 2g, 2i, 2k) as compared to dko IG treated mice (2b, 2d, 2f, 2h, 2j, 2l), respectively.

With exception of diaphragm muscle, there were significantly less inflammatory clusters and collagen percentage in muscle from the IG treated animals in both quadriceps and gastrocnemius (Fig. 2b and f; d and h, respectively) as compared to control animals (Fig. 2a and e; $\mathrm{c}$ and $\mathrm{g}$, respectively), $p<0.05$, with less variability in muscle fibers architecture (better preserved and integrated). The central nucleation index was significantly higher in IG treated mice as compared to controls for quadriceps and gastrocnemius muscles, but not in the diaphragm, $p<0.05$.

\section{IG therapy modulates innate immunity by decreasing dendritic cell activation}

Flow cytometry analyses (Fig. 3) were made for populations of dendritic cells (DCs) obtained from WT ( $n=6$ mice, all being males), dko IG treated ( $n=6$ mice, 4 being males and 2 females) and dko control ( $n=6$ mice, 3 being males and 3 
Fig. 4 IG treated mice show reduced pro-inflammatory cytokines release. ELISA shows IG dampens proinflammatory cytokines secretion in dko treated compared to control mice, data are means $\pm \mathrm{SE}$ of four animals per group (WT, dko control and dko IG) from two independent experiments (n $=2$ mice per group in each one of them), totalizing $\mathrm{n}=12$ mice analyzed $(* p<0.05 ; * * p<0.01$ $* * * p<0.001)$. TNF- $\alpha$ and IL- $1 \beta$ were measured from DCs culture while IFN- $\gamma$ and IL-2 were measured in the DClymphocytes supernatants coculture. For TNF- $\alpha$ and IL- $1 \beta$, white columns represent DCs only and black columns represent DCs activated with muscle antigens
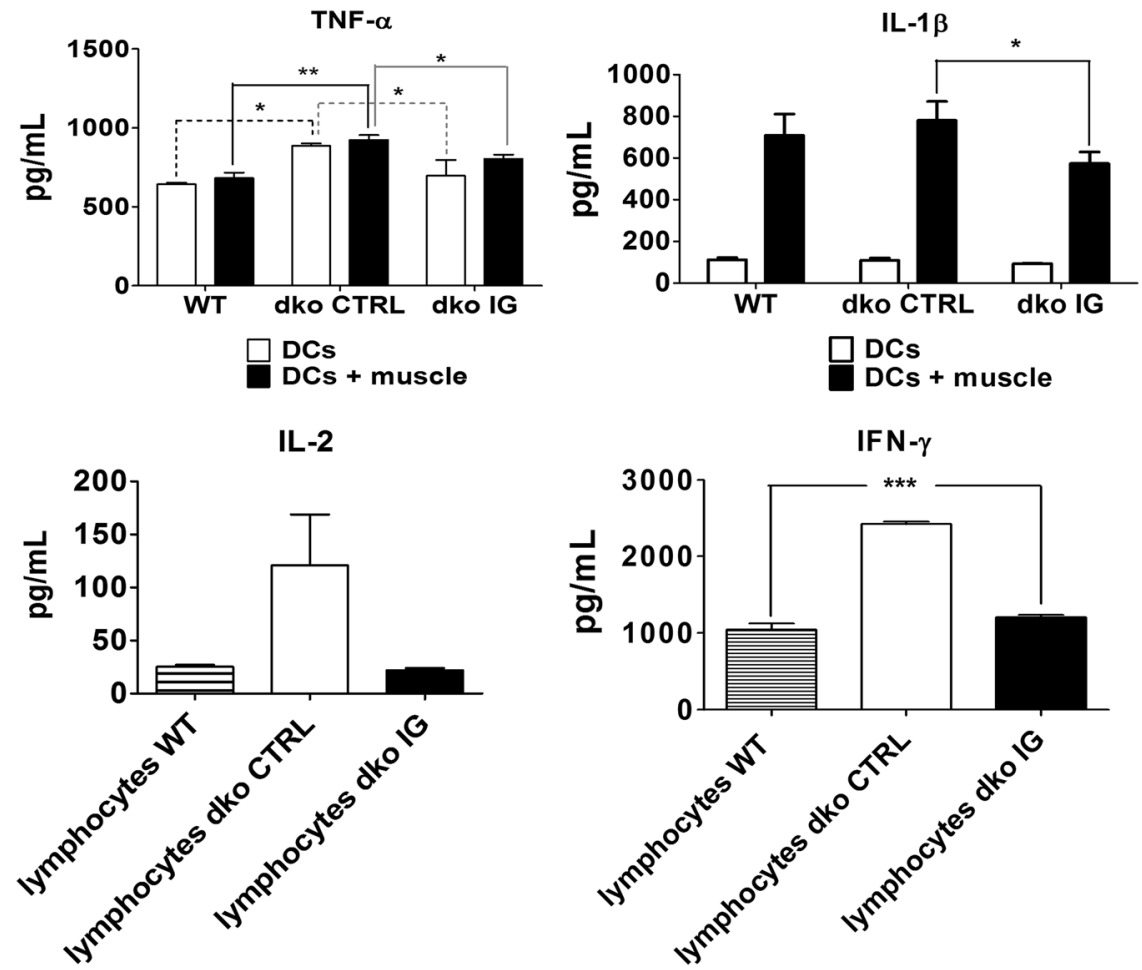

females) groups. DCs were analyzed by their markers of activation, when in contact with dystrophic muscle antigens. MHC, CD86 and CD40 (Fig. 3b) are important costimulatory molecules expressed on antigen presenting cells surface that are essential for mediating $\mathrm{T}$ and $\mathrm{B}$ lymphocytes response, triggering or inhibiting adaptive immunity. There was a statistically significant inhibition of DC activation by muscle antigens in IG treated mice, when compared to controls for all three markers $(p=0.0094$ for $\mathrm{MHC}^{+} ; p=0.0091$ for $\mathrm{CD}^{+}$and $p=0.0213$ for $\mathrm{CD} 40^{+}$). Therefore, flow cytometry results support a role for IG in modulating innate immunity, significantly inhibiting DCs activation in IG treated group.

These observations indicate that dko control mice DCs present increased expression of costimulatory molecules that were all reduced in mice who had received IG therapy, enabling them to recover in part the WT expression pattern.

\section{IG dampens proinflammatory cytokines secretion in dko IG treated mice}

Enzyme-linked immunosorbent assay was performed in both dendritic cells (DCs) and lymphocytes culture supernatants to analyze the cytokines being released by those cells when stimulated by muscle antigens in vitro (Fig. 4). A significant decreased secretion of proinflammatory DCs cytokines IL- $1 \beta$ and TNF- $\alpha$ in the IG treated compared to dko control mice group ( $p=0.0182$ for IL-1 $\beta$ and $p=$ 0.0223 for $\mathrm{TNF}-\alpha$ ) was observed. In lymphocytes, there was a highly significant decrease in IFN- $\gamma$ release in IG treated mice, as compared to controls $(p<0.001)$, recovering almost entirely the WT pattern $(n=12$ mice, 4 per group: 4 males WT; 3 males and 1 female dko IG; 2 males and 2 females dko control). These results suggest that IFN- $\gamma$ is a key proinflammatory cytokine in Duchenne dystrophy immunopathology in vitro.

\section{Discussion}

The therapeutic effect of human polyclonal IgG was recently tested in mdx mice [16]. The authors report beneficial results, ameliorating mice dystrophic phenotype, dampening muscle tissue inflammation and damage, and also improving muscle strength. They also observed motor ability improvement in treated animals as compared to the control groups, together with a decrease in serum creatine kinase levels (CPK) and mRNA expression levels of relevant muscle inflammatory markers. However, the mdx model does not recapitulate the severity of human DMD since these mice are almost asymptomatic and have a normal lifespan [17]. Here we chose the double knockout $\mathrm{mdx} /$ utr- mouse [18], a severely affected murine model for Duchenne muscular dystrophy whose pathology is comparable to human affected patients, despite its different genetic background since they lack utrophin, which is upregulated in DMD boys and mdx mice compared to healthy boys and BL10.C57 mice, respectively. Here we 
show for the first time the clinical beneficial effects of IG injected intraperitoneally in dko (mdx/utr-) mice, decreasing inflammation and increasing significantly their survival rates. Activity rates also were significantly different between the treated vs. the untreated group. However, the possibility that these differences were due to an unequal gender distribution cannot be ruled out since there were more females in the untreated group.

Deficiency in muscle dystrophin causes membrane fragility and cell necrosis, progressive muscle wasting and weakness which results in the severe phenotype of Duchenne muscular dystrophy. Recent therapeutic trials have focused on increasing dystrophin expression through gene therapy (exon skipping) or readthrough approach in DMD patients [2-4]. However, these approaches are applicable for only a subset of patients with specific mutations and the clinical impacts of these current trials are still under evaluation. Furthermore, in DMD patients there are some clusters of revertant fibers expressing dystrophin which have been reported as immunogenic in some patients and more recently in mdx mice [19]. We have previously shown that it is possible to have a functional dystrophic muscle in golden retriever muscular dystrophy (GRMD) dogs and Labrador muscular dystrophy (LRMD) dogs, the animal models closest to human DMD pathology, despite the absence of muscle dystrophin [20-22]. These observations indicate that other therapeutic approaches, such as those involved in immune response mechanisms, not directly related to dystrophin upregulation, but that could act as a complement to dystrophin-based approaches, should be attempted.

As a consequence of muscle cell necrosis, inflammatory cell infiltration occurs in dystrophic muscle. It is believed that there is a predominance of Th1 immune pathways associated with a chronic inflammatory condition mediated by a high population of type M1 macrophages which continuously secrete a number of pro-inflammatory cytokines in dystrophic muscles [6, 7, 23]. When compared to dko controls, IG treated mice had fewer effector responses, with less expression of costimulatory molecules [24] and secretion of proinflammatory cytokines. This would explain the observed diminished muscle fibrosis and histopathological features illustrated in Fig. 2. IG treated mice showed less inflammation and better tissue integrity as compared to dko controls, with less deposition of inflammatory clusters infiltrated within the tissue and collagen fibers. Additionally, there was a significant increase in centrally-nucleated fibers in the quadriceps and gastrocnemius muscles, suggesting a better muscle regeneration in IG treated mice. None of these improvements were observed for the diaphragm muscle, in accordance to previous studies using IG therapy in the $\mathrm{mdx}$ model [16].
Gene therapies aiming to restore dystrophin expression have been the major therapeutic targets of researchers working in the muscular dystrophy field worldwide. Despite many successful trials [2-4], autoreactive T-cell mediated immune responses against dystrophin epitopes have already been reported in human DMD patients [25], GRMD dogs [26] and in mdx mice [27]. These observations reinforce the lack of tolerance of those bearing nonsense DMD mutations who had never been exposed to dystrophin before. As immune responses against dystrophin would unleash severe muscle damage driven mainly by $\mathrm{CD} 8^{+}$cytotoxic $\mathrm{T}$ cells and proinflammatory cytokines such as IFN- $\gamma$, dystrophinbased therapeutic approaches should carefully consider monitoring cellular immune responses that may come along with dystrophin restoration. In this context, immunoglobulin therapy should be a good complement to these approaches aiming to upregulate dystrophin due to its ability to dampen effector immune responses, neutralizing proinflammatory cytokines as well as reducing antigen presentation and consequently, MHC class I-mediated activation of $\mathrm{T}$ cells.

Farini et al. [19], recently reported that the inflammatory state of the muscle tissue seen in Duchenne muscular dystrophy, enriched of proinflammatory cytokines like IFN- $\gamma$ and TNF- $\alpha$, induces the transformation of the myofibers constitutive proteasome into an immunoproteasome, a complex consisting of multiple subunits that play a major role in mediating cellular immunity. This process would increase the antigen presentation via MHC class I, T cell differentiation (with $\mathrm{T}$ cells being activated against revertant dystrophin epitopes) and cytokine release. These authors suggest that the i-proteasome is highly related to DMD pathology and its inhibition could ameliorate the dystrophic phenotype.

Polyclonal IgG immunomodulatory properties are described in literature as capable of attenuating inflammatory processes in clinical conditions such as immunothrombocytopenia (ITP), Kawasaki syndrome (KS), bone marrow transplantation, and Guillain-Barre syndrome (GBS) [8]. Here we show that IG therapy modulates DMD pathophysiology, increasing significantly mice lifespan and motor ability. IG appears to have a modulating role in innate immune pathways, consequently affecting adaptive immunity, since a significant decrease in DCs activation $\left(\mathrm{MHC}^{+}\right.$, $\mathrm{CD} 40^{+}$and $\mathrm{CD}^{+} 6^{+}$) in IG treated mice as compared to controls was observed. ELISA analysis also detected a significant decrease in proinflammatory cytokines TNF- $\alpha$, IL- $1 \beta$ (related to inflammasome activation) and IFN- $\gamma$ in dko IG treated mice, dampening thereby tissue inflammation and reinforcing a role of IG in modulating the severity of muscle wasting in affected animals.

Satellite cells are a population of muscle stem cells committed to myogenesis, activated under mechanical and 
oxidative stresses as well as in inflammatory conditions. In healthy muscles, the pool of satellite cells is maintained as there is a balance between their differentiation into myotubes and their self-renewal [28, 29]. In Duchenne muscular dystrophy, the regenerative capacity of the satellite cells is reduced due to muscle degeneration and inflammatory processes underlying the pathology of the disease. Recent studies suggest a role for the immune system in determining the developmental fate of satellite cells. They show that besides their role in activating classically macrophages towards a M1 phenotype, the pro-inflammatory cytokines IFN- $\gamma$ and TNF- $\alpha$ have a key role in muscle differentiation [28]. IFN- $\gamma$ binding to its receptors on muscle progenitor cells (MPCs) induces expression of the CIITA gene [30], which binds to myogenin (MYOG) preventing muscle differentiation, increasing histone methyltransferase EZH2 activity and silencing important muscle-specific genes due to epigenetic mechanisms $[28,30,31]$. TNF- $\alpha$ binding to its receptors on MPCs has similar effects, as it enhances activity of EZH2, silencing PAX7 and NOTCH1 genes, consequently reducing satellite cell numbers and impairing MPCs differentiation [28, 32]. In this study we show that immunoglobulin therapy decreases levels of both hallmark cytokines for DMD pathology IFN- $\gamma$ and TNF- $\alpha$ in treated mice. This could explain the improved muscle regeneration suggested by an increase in centrally-nucleated fibers seen in histopathological analyses for IG treated mice. That is, the expression of the genes committed to muscle differentiation would be enhanced and in long-term, the balance between differentiation and self-renewal of MPCs might be restored.

Although being an extremely safe procedure, IG therapy may bring some rare adverse reactions in a long-term perspective, most of them related to impurity of the preparation itself. Those reported until the present date include generalized reactions such as headache, fever, chills, myalgia, nausea and vomiting, hypersensitivity and anaphylactic reactions, hemolytic anemia, impaired kidney functions, viral contamination as well as neurological complications [33].

In summary, the results of the present study may open new perspectives for the treatment of DMD, decreasing the progression of the dystrophic process and increasing life expectancy with less side-effects than the currently used glucocorticoids $[5,34]$ that can be very harmful to a subset of patients. Moreover, IG is also a FDA approved therapy already in use for treating patients with autoimmune disorders.

Acknowledgements The collaboration of the following persons is gratefully acknowledged. Maria Neide Ferreira Mascarenhas, Institute of Energy and Nuclear Research (IPEN-SP), for providing the animals for our research; Cláudia Battlehner, University of São Paulo School of Medicine (FMUSP), for her help with histopathological analyses;
Juliana Gomes, Natássia Vieira, Amanda Assoni, Uirá Melo, Luiz Caires, Gerson Kobayashi, Mariz Vainzof, Renata Ishiba, Antonio Ribeiro, Naila Lourenço, Human Genome and Stem-Cell Research Center-Institute of Biosciences of the University of São Paulo (HUG-CELL, IBUSP), for their suggestions and support. The authors thank Baxalta/Shire Brazil for donating Kiovig 10\% (immunoglobulin) used in this study.

Funding This work was supported with grants from FAPESP-CEPID (grant number 2013/08028-1 to M. Zatz and B. G. Nunes), FAPESP (grant number 2015/19435-2 to B. G. Nunes and number 2014/047832 to F.V. Loures) and from National Institute of Science and Technology/National Council for Scientific and Technological Development - INCT/CNPq (grant number 573633/2008-8 to M. Zatz).

\section{Compliance with ethical standards}

Conflict of interest The authors declare that they have no competing interests.

\section{References}

1. Guiraud S, Aartsma-Rus A, Vieira NM, Davies KE, van Ommen GJ, Kunkel LM. The pathogenesis and therapy of muscular dystrophies. Annu Rev Genom Hum Genet. 2015;16:281-308.

2. Nowak KJ, Davies KE. Duchenne muscular dystrophy and dystrophin: pathogenesis and opportunities for treatment. EMBO Rep. 2004;5:872-76.

3. Bengtsson NE, Hall JK, Odom GL, et al. Muscle specific CRISPR/Cas9 dystrophin gene editing ameliorates pathophysiology in a mouse model for Duchenne muscular dystrophy. Nat Commun. 2017;8. https://doi.org/10.1038/ ncomms14454.

4. Van Deutekom JC, Bremmer-Bout M, Janson AA, et al. Antisense-induced exon skipping restores dystrophin expression in DMD patient derived muscle cells. Hum Mol Genet. 2001;10:1547-54.

5. Balaban B, Matthews DJ, Clayton GH, Carry T. Corticosteroid treatment and functional improvement in Duchenne muscular dystrophy: long-term effect. Am J Phys Med Rehabil. 2005;84:843-50.

6. Rosenberg AS, Puig M, Nagaraju K, Hoffman EP, Villalta SA, Rao VA, Wakefield LM, Woodcock J. Immune-mediated pathology in Duchenne muscular dystrophy. Sci Transl Med. 2015;7:299.

7. Spencer MJ, Montecino-Rodriguez E, Dorshkind K, Tidball JG. Helper $(\mathrm{CD} 4+)$ and Cytotoxic (CD8+) $\mathrm{T}$ cells promote the pathology of dystrophin-deficient muscle. Clin Immunol. 2001;98:235-43.

8. Jolles S, Jordan SC, Orange JS, van Schaik IN. Immunoglobulins: current understanding and future directions. Clin Exp Immunol. 2014;178:163-8.

9. Schwab I, Nimmerjahn F. Intravenous immunoglobulin therapy: how does IgG modulate the immune system? Nat Rev Immunol. 2013;13:176-89.

10. Nimmerjahn F, Ravetch JV. The antiinflammatory activity of IgG: the intravenous IgG paradox. J Exp Med. 2007;204:11-5.

11. Dalakas MC. Intravenous immunoglobulin in autoimmune neuromuscular diseases. JAMA. 2004;291:2367-75.

12. Ballow M. The IgG molecule as a biological immune response modifier: mechanisms of action of intravenous immune serum globulin in autoimmune and inflammatory disorders. J Allerg Clin Immunol. 2011;127:315-23. 
13. Lünemann JD, Nimmerjahn F, Dalakas MC. Intravenous immunoglobulin in neurology-mode of action and clinical efficacy. Nat Rev Neurol. 2015;11:80-9.

14. Bayry J, Negi VS, Kaveri SV. Intravenous immunoglobulin therapy in rheumatic diseases. Nat Rev Rheumatol. 2011;7:349-59.

15. Inaba $\mathrm{K}$, Inaba $\mathrm{M}$, Romani $\mathrm{N}$, et al. Generation of large numbers of dendritic cells from mouse bone marrow cultures supplemented with granulocyte/macrophage colony-stimulating factor. J Exp Med. 1992;176:1693-702.

16. Zschüntzsch J, Zhang Y, Klinker F, et al. Treatment with human immunoglobulin $\mathrm{G}$ improves the early disease course in a mouse model of Duchenne muscular dystrophy. J Neurochem. 2016;136:351-62.

17. McGreevy JW, Hakim CH, McIntosh MA, Duan D. Animal models of Duchenne muscular dystrophy: from basic mechanisms to gene therapy. Dis Model Mech. 2015;8:195-213.

18. Deconinck AE, Rafael JA, Skinner JA, et al. Utrophin-dystrophindeficient mice as a model for Duchenne muscular dystrophy. Cell. 1997;90:717-27.

19. Farini A, Sitzia C, Cassani B, et al. Therapeutic potential of immunoproteasome inhibition in Duchenne muscular dystrophy. Mol Ther. 2016;24:1898-1912.

20. Zatz M, Vieira NM, Zucconi E, et al. A normal life without muscle dystrophin. Neuromuscul Disord. 2015;25:371-4.

21. Vieira NM, Guo LT, Estrela E, Kunkel LM, Zatz M, Shelton GD. Muscular dystrophy in a family of labrador retrievers with no muscle dystrophin and a mild phenotype. Neuromuscul Disord. 2015;25:363-70.

22. Vieira NM, Elvers I, Alexander MS, et al. Jagged 1 rescues the duchenne muscular dystrophy phenotype. Cell. 2015;163:1204-13.

23. Villalta SA, Rosenberg AS, Bluestone JA. The immune system in Duchenne muscular dystrophy: friend or foe. Rare Dis. 2015;3. https://doi.org/10.1080/21675511.2015.1010966.
24. Bayry J, Lacroix-Desmazes S, Carbonneil C, et al. Inhibition of maturation and function of dendritic cells by intravenous immunoglobulin. Blood. 2003;101:758-65.

25. Mendell JR, Campbell K, Rodino-Klapac L, et al. Dystrophin immunity in Duchenne's muscular dystrophy. N Engl J Med. 2010;362:1429-37.

26. Sitzia C, Farini A, Jardim L, et al. Adaptive immune response impairs the efficacy of autologous transplantation of engineered stem cells in dystrophic dogs. Mol Ther. 2016;24:1949-64.

27. Ferrer A, Wells KE, Wells DJ. Immune responses to dystrophin: implications for gene therapy of Duchenne muscular dystrophy. Gene Ther. 2000;7:1439-46.

28. Tidball JG. Regulation of muscle growth and regeneration by the immune system. Nat Rev Immunol. 2017;17:165-78.

29. Tidball JG, Villalta SA. Regulatory interactions between muscle and the immune system during muscle regeneration. Am J Physiol Regul Integr Comp Physiol. 2010;298:1173-87.

30. Londhe P, Davie JK. $\boldsymbol{\gamma}$-Interferon modulates myogenesis through the major histocompatibility complex class II transactivator, CIITA. Mol Cell Biol. 2011;31:2854-66.

31. Londhe P, Davie JK. Interferon- $\gamma$ resets muscle cell fate by stimulating the sequential recruitment of JARID2 and PRC2 to promoters to repress myogenesis. Sci Signal. 2013;6:ra107.

32. Acharyya S, Sharma SM, Cheng AS, et al. TNF inhibits NOTCH1 in skeletal muscle cells by EZH2 and DNA methylation mediated repression: implications in Duchenne muscular dystrophy. PLoS One. 2010;5:e12479. https://doi.org/10.1371/journal.pone. 0012479

33. Duhem C, Dicato MA, Ries F. Side-effects of intravenous immune globulins. Clin Exp Immunol. 1994;97:79-83.

34. Godeuau B, Chevret S, Varet B, et al. Intravenous immunoglobulin or high-dose methylprednisolone, with or without oral prednisone, for adults with untreated severe autoimmune thrombocytopenic purpura: a randomised, multicentre trial. Lancet. 2002;359:23-9. 